\title{
Hipotermia intravascular inducida en el manejo de la hipertensión intracraneana en insuficiencia hepática aguda. Caso clínico
}

\author{
Luis Castillo $\mathbf{F}^{\mathbf{1}}$, Cristián Pérez $\mathbf{R}^{\mathbf{1}}$, Carolina Ruiz $\mathbf{B}^{\mathbf{1}}$, \\ G uillermo Bugedo $\mathrm{T}^{1}$, G lenn Hernández $\mathrm{P}^{1}$, \\ Jorge Martínez $\mathrm{C}^{2}$, Nicolás Jarufe $\mathrm{C}^{2}$, Rosa Pérez $\mathrm{A}^{3}$, \\ Patricio Mellado T ${ }^{4}$, Pilar Domínguez ${ }^{3 a}$. \\ Intravascular hypothermia \\ for the management of intracranial \\ hypertension in acute liver failure. \\ Case report
}

Acute liver failure has a mortality rate in excess of $80 \%$. Most deaths are attributed to brain edema with intracranial hypertension and herniation of structures, where ammonium plays a major role in its generation. We report an 18 year-old female with a fulminant hepatic failure caused by virus A infection. The patient developed a profound sopor and required mechanical ventilation. A CT scan showed the presence of brain edema and intracranial hypertension. A Raudemic ${ }^{\circledR}$ catheter was inserted to measure intracranial pressure and brain temperature. Intracranial hypertension became refractory and intravascular hypothermia was started, reducing brain temperature to $33^{\circ} \mathrm{C}$. Seventy two hours later, a liver transplantation was performed. After testing graft perfusion, rewarming was started, completing 122 hours of hypothermia at $33^{\circ} \mathrm{C}$. The patient was discharged in good conditions after 69 days of hospitalization (Rev Méd Chile 2009; 137: 801-6).

(Key words: Brain edema; Hypothermia; Liver failure, acute)
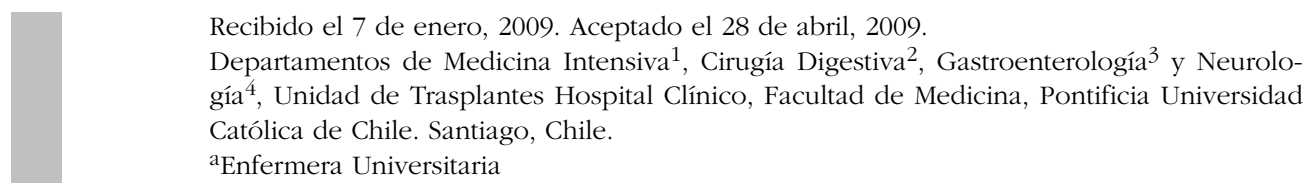
gía $^{4}$, Unidad de Trasplantes Hospital Clínico, Facultad de Medicina, Pontificia Universidad Católica de Chile. Santiago, Chile.

${ }^{a}$ Enfermera Universitaria

\begin{abstract}
$\mathrm{L}$ a insuficiencia hepática aguda (IHA) caracterizada por el desarrollo de encefalopatía, dentro de las 4 semanas siguientes a la aparición de
\end{abstract}

$\overline{\text { Correspondencia a: Dr. Luis Castillo F. Marcoleta 367. De- }}$ partamento de Medicina Intensiva, Hospital Clínico Pontificia Universidad Católica de Chile.

E mail: castillo@med.puc.cl ictericia en ausencia de patología hepática previa, se acompaña de disfunción metabólica, hepatocelular y de síntesis con coagulopatía severa ${ }^{1-2}$. La mortalidad fluctúa entre $60 \%$ y $80 \%$ en ausencia de trasplante hepático, siendo éste el único tratamiento para revertir la función hepática ${ }^{2-5}$.

El edema encefálico con hipertensión intracraneana (HIC) y herniación de estructuras es el 
responsable de la mortalidad hasta en 90\% de los casos $^{6-7}$. Su reconocimiento y tratamiento precoz es indispensable para disminuir la mortalidad, prevenir lesiones neurológicas irreversibles e incrementar el tiempo de espera de donación de órganos.

Se desconoce el porcentaje de pacientes con IHA que desarrollan HIC, siendo la disfunción neurológica un factor de riesgo para su desarrollo, incrementándose a medida que progresa la encefalopatía de 25\% a 35\% en el grado III hasta 65\% a $75 \%$ en el grado $\mathrm{IV}^{8}$.

Presentamos un caso de IHA con HIC severa refractaria, en el que se indujo hipotermia intravascular como mecanismo de neuroprotección y control.

\section{Caso Clínico}

Mujer de 18 años sana, dos semanas previas a su ingreso presentó ictericia, astenia, adinamia y compromiso de conciencia progresivo. Al ingreso hubo elevación de transaminasas, gamaglutamiltranspeptidasas, aumento de INR y disfunción renal (Figura 1). No hubo antecedentes de consumo de fármacos y los marcadores virales e inmunológicos fueron negativos exceptuando, un título de intensidad menor para virus hepatitis A.

Evolucionó rápidamente a sopor profundo, requirió ventilación mecánica invasiva y estudio con tomografía computarizada de encéfalo (TC) que evidenció edema encefálico con elementos sugerentes de HIC (Figura 2). La monitorización electroencefalográfica mostró ritmos lentos y simétricos bilaterales. El INR inicial de 5,0 se corrigió a 1,6 con $40 \mathrm{ug} / \mathrm{kg}$ de Factor VII activado recombinante (rFVIIa) previo a la instalación de catéter intraparenquimatoso Raudemic ${ }^{\circledR}$ para medición de la presión intracraneana (PIC) y temperatura encefálica. Los valores iniciales de PIC fluctuaron entre 20 y $25 \mathrm{mmHg}$, con temperatura cerebral de $37^{\circ} \mathrm{C}$ (Figura 1). El Doppler transcraneano determinó velocidad sistólica e índice de pulsatibilidad normales; siendo enlistada para trasplante hepático de acuerdo a los criterios del King's College ${ }^{8}$.

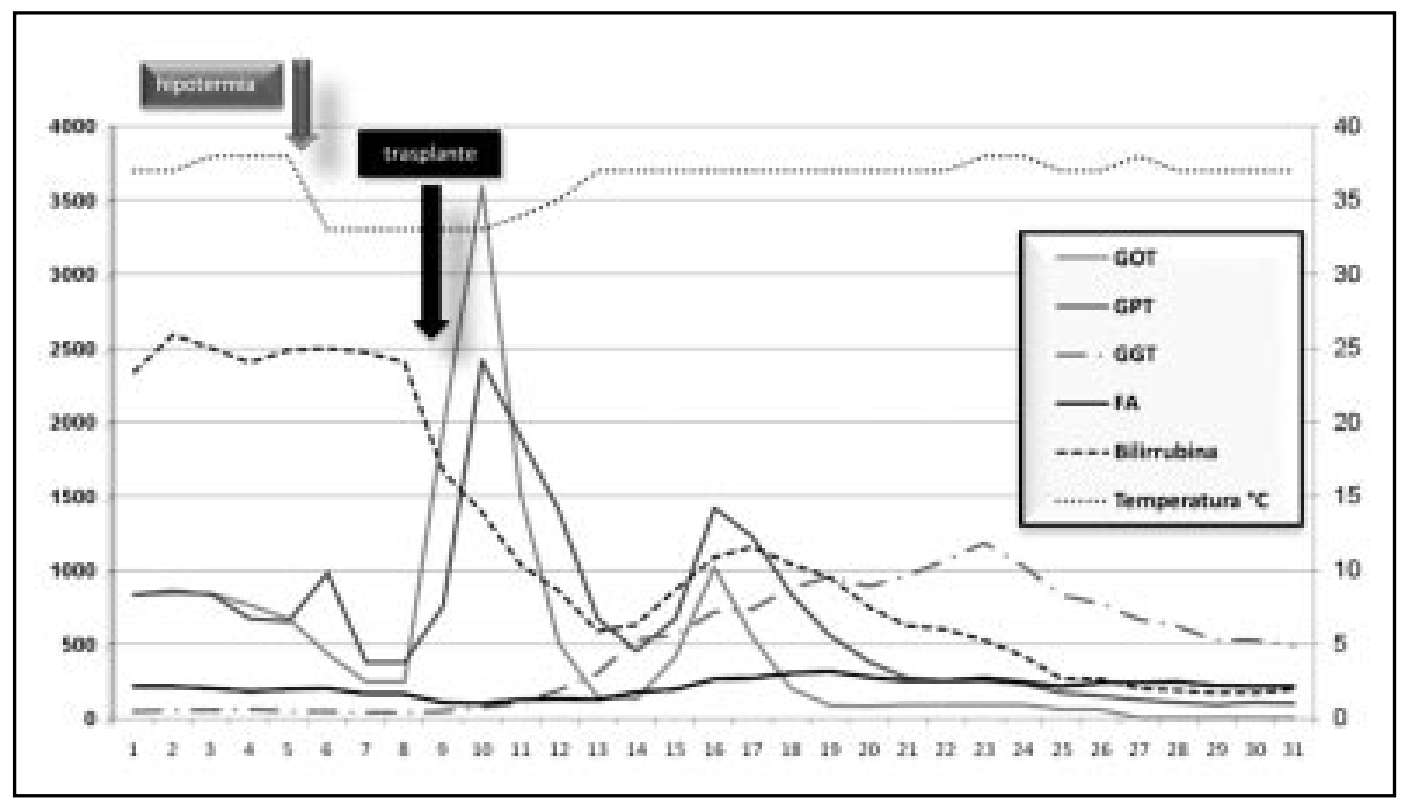

Figura 1. Muestra en relación al eje principal, la evolución de glutamato-oxalacetato transaminasa (GOT), glutamato-piruvato transaminasa (GPT), gamma-glutamil transpeptidasa (GGT), fosfatasa alcalina (FA), mientras que en el eje secundario se grafican los valores de bilirrubina total y temperatura expresada en grados centígrados $\left({ }^{\circ} \mathrm{C}\right)$. Se señala con flecha gris el inicio de hipotermia y con flecha negra el día del trasplante. 


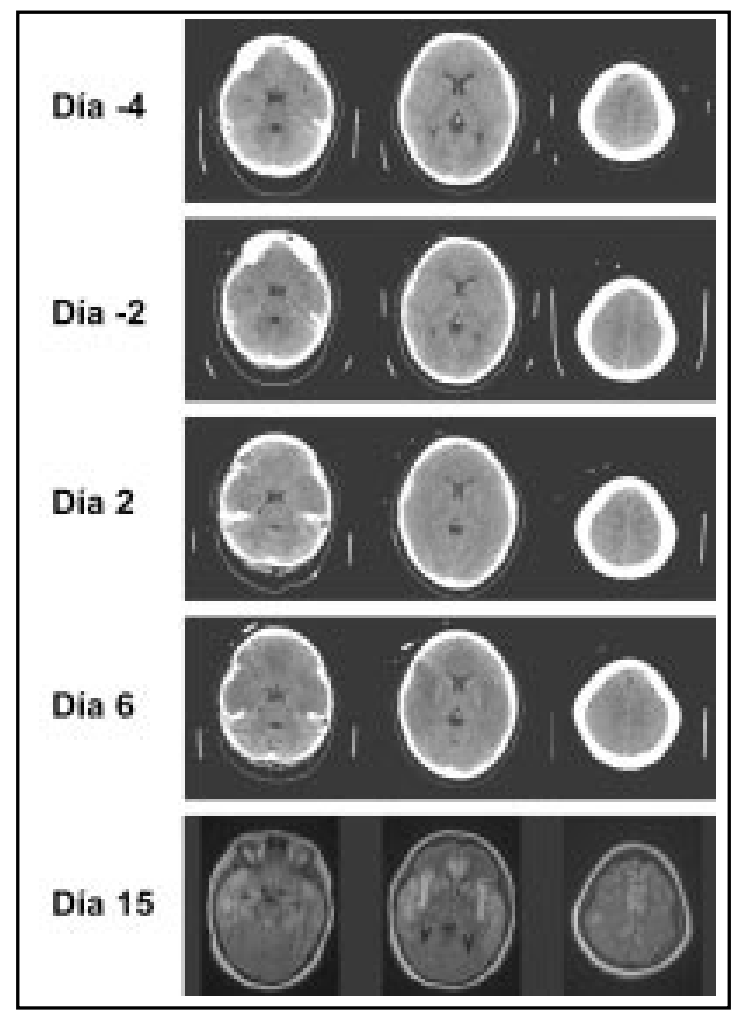

Figura 2. Muestra la evolución de imágenes de TC. En los cuadros a la derecha se detalla la temporalidad respecto al día del trasplante hepático. Se aprecia diferentes grados de edema encefálico, con borramiento de los surcos. Abajo la RNM se observan extensas lesiones corticales distribuidas en ambos hemisferios en regiones frontales mesiales, corteza insular, frontal inferior y temporal bilateral y parietal izquierda, hiperintensas en difusión y TR largo.

La HIC se tornó refractaria a medidas de primera línea (Figura 3), iniciándose hipotermia intravascular mediante catéter tipo ICY ${ }^{\circledR}$ vía femoral con máquina Alsius CoolGard ${ }^{\circledR}$, disminuyendo la temperatura encefálica a $33^{\circ} \mathrm{C}$. Recibió sedación con opiáceos (Fentanyl) e infusión continua de relajante muscular (vecuronium) para prevenir escalofríos; en 3,5 h se logró la meta de $33^{\circ} \mathrm{C}$. A las $72 \mathrm{~h}$, se le ofreció un injerto hepático de donante cadavérico y se realizó un trasplante ortotópico bajo hipotermia intravascular activa. Posteriormente en la unidad de cuidados intensi- vos, y luego de comprobar la perfusión del injerto, se reinició recalentamiento lento controlado alcanzando la normotermia a las 76 h con mejoría continua en las pruebas de función hepática (Figura 1), completando de esta manera 122 h de hipotermia a $33^{\circ} \mathrm{C}$. Desarrolló mioneuropatía del paciente crítico y permaneció en ventilación mecánica durante 15 días, requiriendo de traqueostomía percutánea para facilitar el destete del ventilador mecánico.

Luego de un despertar lento, se retiró la cánula de traqueostomía al día 37. Recibió el alta luego de 69 días de hospitalización en buenas condiciones generales, nutritivas, neurológicas y ligada a un programa de rehabilitación física. Actualmente asiste a sus estudios secundarios y se mantiene en control ambulatorio.

\section{DisCUSIÓN}

El desarrollo de edema encefálico con HIC en la IHA tiene un pronóstico ominoso, siendo el trasplante hepático la única terapia efectiva de tratamiento $9-11$. Como puente a éste, varias medi-

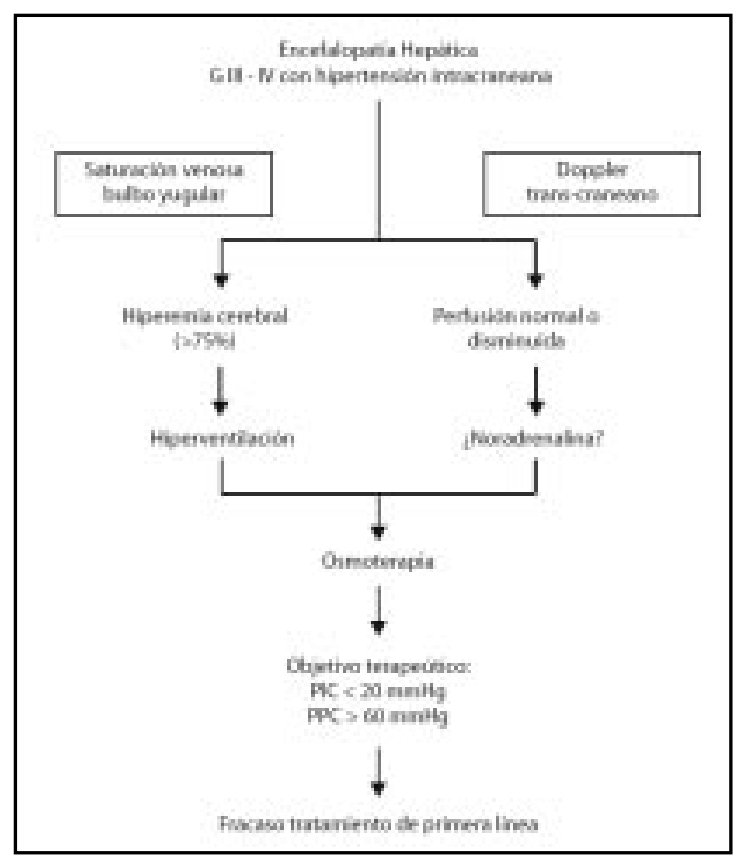

Figura 3. Encefalopatía hepática. G III-IV con hipertensión intracraneana (figura elaborada por los autores). 
das se utilizan para mitigar el edema encefálico.

Para establecer la existencia y magnitud de la HIC se requiere instalar un dispositivo de monitorización continua, siendo imprescindible revertir la coagulopatía asociada. El rFVIIa se ha usado con este fin, con resultados aceptables como en el caso de nuestra paciente.

El Doppler transcraneano, ha mostrado también una buena correlación entre el índice de pulsatibilidad y PIC; mientras que la velocidad sistólica en la arteria cerebral media se ha usado como estimador indirecto del flujo sanguíneo encefálico (FSE).

En el tratamiento de la HIC en la IHA se ha planteado un manejo de primera línea que incluye elevación y posicionamiento de la cabeza, sedación con agentes neuroprotectores, hiperventilación y administración de soluciones hipertónicas (Figura 3) y para los casos de HIC refractaria uno de segunda línea que incluye hipotermia y sistemas de soporte hepático 7,9,11 (Figura 4).

En el caso presentado, utilizamos hipotermia controlada como terapia de segunda línea, sobre lo cual revisamos algunas consideraciones.

Hipotermia moderada (32-35ํㅡ). El amonio juega un papel fundamental en la producción de edema encefálico, su detoxificación se basa casi totalmente en la acción de la glutamin sintetasa en los astrocitos. Por su parte, el efecto osmótico por el acúmulo de glutamina explicaría el edema astrocitario selectivo.

La hipotermia ha mostrado retardar y disminuir el edema encefálico y la aparición de encefalopatía ${ }^{12}$. La producción de amonio intestinal disminuye y mejora la excreción renal, reduciendo sus concentraciones arteriales y en líquido cefalorraquídeo ${ }^{13-14}$. Osmolitos y solutos como glucosa, alanina y lactato que también se encuentran alterados, disminuyen; éstos dos últimos correlacionados con el grado de encefalopatía ${ }^{15-16}$.

Mediante microdiálisis cerebral se han comprobado incrementos en las concentraciones extracelulares de aminoácidos aromáticos y neurotransmisores, algunos de los cuales fueron atenuados mediante inducción de hipotermia ${ }^{17}$.

En modelos animales, la producción intestinal, captación y concentración arterial y encefálica de glutamato y amonio disminuyen; así como el edema causado por glutamato y lactato. Hay restauración de la autorregulación cerebral con disminución de la hiperemia y del consumo de glucosa y oxígeno a nivel encefálico ${ }^{12,14,18}$.

Sin embargo, quizá el efecto neuroprotector más importante de la hipotermia sea sobre el consumo de oxígeno, al disminuir los requerimientos energéticos en alrededor de $7 \%$ por cada grado de disminución en la temperatura central. Adicionalmente, a nivel celular y de neurotransmisión disminuye la actividad electrofisiológica aun en condiciones de silencio electroencefalográfico ${ }^{18}$. El metabolismo de la glucosa también disminuye con la hipotermia, disminuyendo por ende la concentración de piruvato y lactato, relacionados con el desarrollo de edema encefálico.

La hipotermia favorece además la estabilización iónica de membranas, las cuales pierden la capacidad de regulación del agua intracelular causando edema, degradación de lípidos, proteólisis, disfunción mitocondrial y producción de radicales libres?.

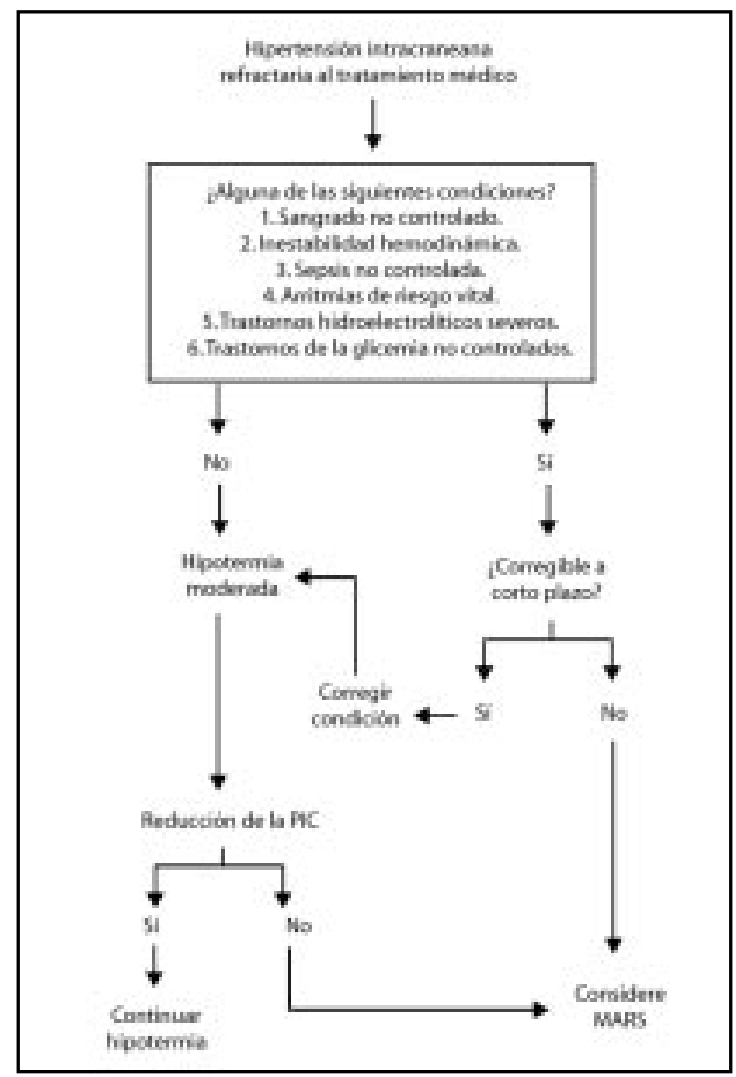

Figura 4. Hipertensión intracraneana refractaria al tratamiento médico (figura elaborada por los autores). 
Experimentalmente la hipotermia disminuye rápida y persistentemente la PIC, el FSE, las presiones de perfusión encefálica y los requerimientos de drogas vasoactivas, incluso durante el trasplante hepático, por lo que se recomienda mantenerla durante el procedimiento. No existe evidencia de cambio en la mortalidad con su uso ${ }^{19}$.

Su recomendación está limitada debido a la falta de estudios aleatorizados en IHA, así como a la existencia de eventos adversos derivados de su uso, se recomienda además cautela en su uso bajo condiciones como sangrado no controlado, inestabilidad hemodinámica, sepsis no controlada, arritmias de riesgo vital, trastornos hidroelectrolíticos severos y trastornos de la glicemia de difícil manejo.

¿Cómo realizar hipotermia? Técnicamente, existen tres fases en su aplicación: inducción, intentando alcanzar el objetivo de temperatura en el menor tiempo posible, mantención en la que se prolonga la constante térmica deseada, y una fase de recalentamiento lenta y controlada.

Las técnicas utilizadas son:

1. Hipotermia centrífuga por enfriamiento cutáneo con bolsas de hielo. Económica, de fácil implementación y carente de complicaciones técnicas; pero lenta y con dificultad en el control y regulación de la velocidad de enfriamiento, mantenimiento y recalentamiento.

2. Hipotermia centrífuga con cobija de aire circulante o soluciones salinas frías. No invasivo; de uso masivo por su bajo costo y fácil implementación, ineficiente en las tres fases tanto en tasas como en velocidades.

3. Hipotermia intravascular mediante catéteres. Muy eficientes en las fases de inducción,

\section{REFERENCIAS}

1. Detry O, De Roover A, Honoré P, Meurisse M. Brain edema and intracranial hypertension in fulminant hepatic failure: Pathophysiology and management. World J Gastroenterol 2006; 12: 7405-12.

2. Gimson AE. Fulminant and late onset hepatic failure. BJA 1996; 77: 90-8.

3. LAI WK, MurPhy N. Management of acute liver failure. Continuing Education in Anaesthesia. Crit Care \& Pain 2004; 4: 40-3. mantención y recalentamiento, estos dispositivos han ganado terreno en los últimos años.

4. Enfriamiento a través de la membrana pulmonar con intercambiador de calor inspiratorio. De escaso uso y con alta frecuencia de complicaciones pulmonares.

El tiempo óptimo de la fase de mantención se desconoce, variando entre 12 y $72 \mathrm{~h}$, dependiendo de la patología a tratar. En el caso de la HIC por IHA, al tratarse de una noxa prolongada requiere de una fase extendida, por lo que debería mantenerse mientras se resuelve la patología hepática y no existan contraindicaciones para su uso.

En nuestro caso la hipotermia sistémica se indujo a través del sistema intravascular controlado con catéter femoral de tres lúmenes y conexión a dispositivo Alsiuss ${ }^{\circledR}$. Estos catéteres constan de 3 balones para la instalación femoral y dos balones para la instalación subclavia o yugular. Por ellos circula solución salina enfriada a 2 ó $4^{\circ} \mathrm{C}$ por una solución congelante en el dispositivo externo.

El control de la temperatura en la fase de mantención es guiado por un sensor instalado en el lecho vesical mediante una sonda, mientras que la fase de recalentamiento es regulable y controlable.

\section{CONClusión}

Se presenta el primer caso reportado de una paciente con IHA, que evolucionó con HIC refractaria a tratamientos de primera línea en quien se utilizó hipotermia intravascular como medida coadyuvante antes, durante y después del trasplante hepático evolucionando de forma exitosa.

4. Raghavan M, MariK PE. Therapy of Intracranial Hypertension in Patients With Fulminant Hepatic Failure. Neurocrit Care 2006; 4: 179-89.

5. O'Grady JG. Acute liver failure. Postgrad Med J 2005; 81: $148-54$.

6. Jalan R, Rose C. Hypothermia in Acute Liver Failure. Metabolic Brain Disease 2004; 19: 215-21.

7. Jalan R, Damink S, Deutz N, Lee A, Hayes P. Moderate hypothermia for uncontrolled intracranial hypertension in acute liver failure. Lancet 1999; 354: 1164-8. 
8. O'Grady JG, Alexander GJ, Hayllar KM, Williams R. Early indicators of prognosis in fulminant hepatic failure. Gastroenterology 1989; 97: 439-45.

9. Vaquero J, Rose C, Butterworth R. Keeping cool in acute liver failure: Rationale for the use of mild hypothermia. J of Hepatology 2005; 43: 1067-77.

10. De Rave S, Hugo W. Heterotopic and orthotopic liver transplantation in man. Liver Transplantation 2005; 11: 396-401.

11. Hoofnagle JH, Carithers RL Jr, Shapiro C, Ascher N. Fulminant hepatic failure: summary of a workshop. Hepatology 1995; 21: 240-52.

12. Jalan R, Damink SW, Deutz ne, Hayes PC, Lee A. Moderate hypothermia in patients with acute liver failure and uncontrolled intracranial hypertension. Gastroenterology 2004; 127: 1338-46.

13. Córdoba J, Crespin J, Gottstein J, Blei AT. Mild hypothermia modifies ammonia-induced brain ede$\mathrm{ma}$ in rats after portacaval anastomosis. Gastroenterology 1999; 116: 686-93.

14. LaRSen FS, Wendon J. Brain edema in liver failure: basic physiologic principles and management. Liver Transpl 2002; 8: 983-9.
15. Swain M, Butterworth RF, Blei AT. Ammonia and related aminoacids in the pathogenesis of brain edema in acute ischemic liver failure in rats. Hepatology 1992; 15: 449-53.

16. Rose C, Michalak A, Rambaldi A, Chatauret N, Pannunzio M, Butterworth RF. Mild hypothermia delays the onset of coma and prevents brain edema and extracellular brain glutamate accumulation in rats with acute liver failure. Hepatology 2000; 31: 872-7.

17. Zwingmann C, Chatauret N, Rose C, Leibfritz D, Butterworth R. Selective alterations of brain osmolytes in acute liver failure: protective effect of mild hypothermia. Brain Research 2004; 999: 118-23.

18. Wendon JA, Harrison PM, Keays R, Williams R. Cerebral blood flow and metabolism in fulminant liver failure. Hepatology 1994; 19: 1407-13.

19. Vaquero J, Butterworth RF. Mechanisms of brain edema in acute liver failure and impact of novel therapeutic interventions. Neurological Research 2007; 29: 683-90. 